\title{
Randomized PHEV Charging Under Distribution Grid Constraints
}

\author{
Kan Zhou, and Lin Cai, Senior Member, IEEE
}

\begin{abstract}
Plug-in Hybrid Electrical Vehicles (PHEV) are promising to improve energy efficiency and environment friendliness. However, without proper control, their charging will cause harmful impact on the power distribution grid, including load congestion and voltage drop. Instead of using centralized optimization which may need accurate predictions on key parameters, in this paper, a new decentralized random access framework is introduced to schedule the PHEV charging. The proposed distributed solution does not need accurate predictions and can be executed online. Simulation on a semi-urban residential medium voltage grid shows that our algorithm can effectively provide demand response to protect the distribution grid from bus congestion and voltage drop, and also improve its efficiency. Most importantly, this algorithm is simple to deploy.
\end{abstract}

Index Terms-Decentralized/Randomized PHEV charging, Demand Response, Distribution Grid, Smart Grid

\section{INTRODUCTION}

Plug-in hybrid electric vehicle (PHEV) becomes increasingly popular. The energy department of USA estimates that more than one million PHEVs will be sold by the end of 2015. In addition to its environment friendliness, the adoption of a large number of PHEVs will exert great pressure on the current power grid due to its high power demand [2]. As a result, appropriate actions are needed to eliminate any possible harmful impact, which sparks numerous research efforts.

The previous work mainly focused on the grid constraints at the transport and high-voltage distribution grid [1], [2]. Recent research started to pay attention to the distribution grid. The two most common problems in the distribution grid are bus congestion and voltage drop. As discussed in the related work section, existing approaches mainly focus on centralized optimization technologies which need accurate predictions and may be difficult to solve within a short time period given a large grid size. In the low voltage grid, some centralized lightweight control algorithms were proposed, but they may not be easily extended to the whole distribution grid with a large population and high PHEV penetration.

According to our literature survey, there still lacks of a distributed scheduling approach for supporting a high PHEV penetration rate and considering the common grid constraints. In this paper, we propose a framework to regulate PHEV charging by considering the bus load congestion and voltage drop problems in the distribution grid. Different from the existing algorithms, our algorithm is decentralized with a low complexity. No complex optimization problem needs to be solved. And it does not rely on any accurate prediction on load or PHEV arrival time and can be executed in realtime. In addition, our approach takes the delay constraints of PHEV charging into consideration. Finally, it can be extended to include other elastic loads to provide automatic demand response to protect the power grid and improve its efficiency.

The main contributions of this paper are three-fold. First, a decentralized algorithm is introduced, which can efficiently avoid bus congestion and large voltage drop in the distribution grid with charging PHEVs. The smart agents schedule the PHEV charging independently based on the received information of the current grid status from a control center. Second, we analyze the performance of our algorithm and derive the system capacity. Finally, extensive simulation with real data from National House Hold Travel Survey 2009 [11] and the RELOAD database [12] from national energy modeling system are conducted to evaluate the performance of the proposed algorithm on a typical resident area distribution grid [5].

The rest of the paper is organized as follows. A detailed discussion of related work is given in Section II. Section III provides the modeling of current load and PHEV charging profiles, and introduces the grid architecture. Then the problem is formulated in Section IV. A general description of the proposed framework is given in Section V. Then we introduce the detailed algorithm design in Section VI. The performance of the proposed algorithm is analyzed in Section VII. Performance evaluation by simulation is given in Section VIII, followed by concluding remarks and future research issues in Section IX.

\section{RELATED WORK}

J. Taylor et. al demonstrated that a high PHEV penetration rate would result in loads exceeding current bus capacity through simulation based on a real data model [3]. In [4], the authors used load flow analysis to show the impact of PHEV on the distribution grid. J. A. et. al investigated the impact of PHEV charging on medium voltage grid, considering the bus load congestion, and voltage drop problems [5].

To solve the problems listed above in the distribution grid, O. Sundstrom et. al proposed a centralized approach aiming to reach minimum charging cost using an optimization technology. Their model concerns both bus congestion and voltage drop problems in the medium voltage grid [6], [7]. Richardson et. al formulated and solved an optimization problem to maximize the energy delivered to all electrical vehicles (EVs) within a certain period of time [27]. Transformer overload and voltage drop of a low-voltage transmission grid are considered, assuming the charging rate of each EV can be adjusted continuously. M. D. Galus et. al proposed a hierarchic PHEV scheduling algorithm based on model predictive control and game theory in [22], aiming to avoid transformer overload. In [26], the PHEV charging process can respond to frequency 


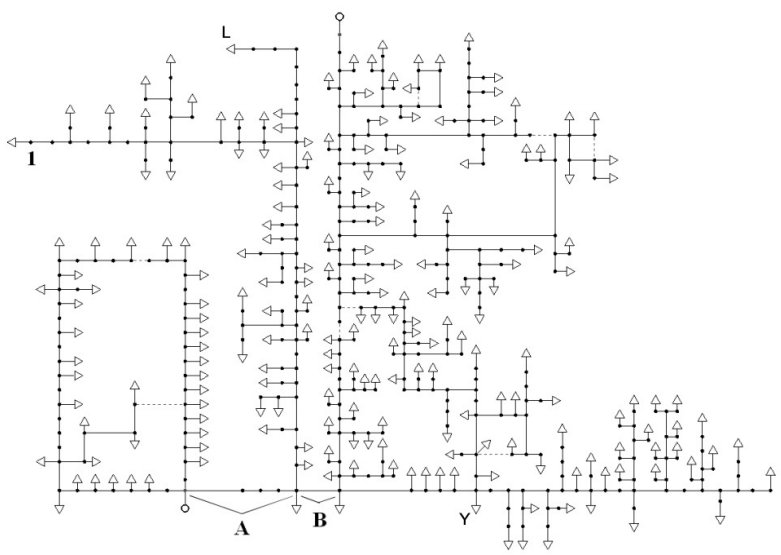

Fig. 1. Grid Architecture [5]

and voltage deviations detected locally. [24] uses stochastic programming to minimize the power losses of the distribution grid. Sortomme et al discussed a method to reduce computation time of minimizing the impacts of PHEV charging on the distribution system's losses in [25]. In [9], each house is assigned an upper bound for power consumption. After reaching this bound, a centralized controller will shut down some devices according to a predefined priority. In [10], a dynamic upper bound for all the houses based on the rule that the original peak demand without PHEV charging is maintained.

The centralized control used in the previous work may not be easily extended to a large-scale distribution grid with high PHEV penetration, as perfect prediction information may not be available, and the computation time to obtain the control actions by solving complex optimization problems may be long. Also, some users may not want their applications being controlled by others due to privacy reasons. These issues motivate us to design a distributed algorithm which does not need accurate prediction on users' behavior and future load information [18]. How to design such a distributed algorithm that can be executed online to solve common distribution grid problems is still an open issue.

\section{System MODEL}

In this section, the topology of the distribution grid, the load profile and PHEV charging patterns are introduced. The PHEV charging profile is modeled based on the data from National House Hold Travel Survey 2009 using a stochastic approach.

\section{A. Medium Voltage Grid in Our Case Study}

Fig. 1 shows a typical residential area distribution grid in Portugal [5], corresponding to a semi-urban $15 \mathrm{kV}$ medium voltage grid in a residential area. The triangular shapes in this figure represent the Medium Voltage to Low Voltage (MV/LV) transformers. Each transformer serves 4 neighborhoods including 10 people on average. ${ }^{1}$

\footnotetext{
${ }^{1}$ Our algorithm can be scalable to support more people. However the simulation time of the bench-mark algorithm using exhaustive search will be much longer.
}

TABLE I

PHEV TYPES AND THEIR KEY PARAMETERS

\begin{tabular}{|c|c|c|c|}
\hline PHEV Types & Battery Capacity & Max Range & Market Share \\
\hline Auto & $24 \mathrm{kWh}$ & 73 miles & $49.9 \%$ \\
\hline SUV & $37.6 \mathrm{kWh}$ & 80 miles & $19.4 \%$ \\
\hline Pickup & $30 \mathrm{kWh}$ & 55 miles & $17.8 \%$ \\
\hline Van (and others) & $36 \mathrm{kWh}$ & 60 miles & $12.9 \%$ \\
\hline
\end{tabular}

Similar to [5]-[7], this medium voltage grid is explored using a radial configuration. All the dashed branches are considered to be open. The two round shapes in Fig. 1 represent the feeding points. As discussed in [5], this grid may experience two main problems: the buses near the feeding points may reach a high congestion level while the far away ones may encounter the voltage drop problem. ${ }^{2}$

\section{B. Distribution Grid Load}

The total load profile for the medium voltage grid used is from [14] which is based on the hourly residential load curves of an average household from the RELOAD database [12] and interpolated using the approach described in [8]. The hourly data is interpolated into the load curve which consists of 1440 minutes of a day.

The load curve consists of 2 different types of residential loads, including both critical loads and elastic loads. Critical loads refer to those that cannot be delayed, such as for cooking and lighting. Elastic loads can be delayed, like for cooling/heating. In this paper, we assume all the loads except PHEV charging to be critical loads for simplicity. In other words, only PHEV charging is controllable and delayable, although, if needed, other elastic loads can also take part in the demand response process using the proposed framework.

\section{PHEV Charging Modeling}

To obtain the PHEV charging profile, we need to know the driving habits, PHEV types and etc. We use the data from National Household Travel Survey (NHTS) 2009 under the assumption that PHEV owners' preferences to vehicle types and their driving behaviors will be similar to the conventional vehicle owners'. From the NHTS report, vehicles can be classified into Auto, Sport Utility Vehicle (SUV), Pick-up trucks and Van. Their key parameters including the estimated market share are shown in Table I.

In this paper, the Monte Carlo method is used to simulate the daily driving distance for each PHEV by using the driving data from [19], so the state of charge (SOC) of each vehicle can be determined when they arrive home based on the battery profile and the maximum driving range of that PHEV. Similar to [10], we further assume that the arriving and departure time of PHEVs follows a normal distribution with the mean of $6 \mathrm{pm}$ and $7 \mathrm{am}$ respectively and a standard deviation of $1 \mathrm{hr}$, respectively. As our focus is to deal with the impact of PHEV charging during peak time on a residential distribution grid, peak hours are included in the charging period.

\footnotetext{
${ }^{2}$ Note that the load used in [5] is different from that in this paper although the same grid topology is used.
} 
In addition to the driving patterns, charging power from [2], [20], [23] is also used to build the PHEV charging model. In this paper, the charging power of $1.4 \mathrm{~kW}, 2 \mathrm{~kW}$ and $6 \mathrm{~kW}$ are chosen with probability $0.45,0.45$ and 0.1 , respectively.

Since there are $207 \mathrm{MV} / \mathrm{LV}$ transformers in the target grid and each transformer severs 10 people, the population size is 2070. From the data of Major Travel Indicators of 2009 [11], the vehicle ownership ratio is $74.4 \%$. Therefore, the number of vehicles is 1540 .

\section{Problem Formulation}

We consider a discrete-time system, i.e., time is divided into slots with a constant duration. We also set the slot duration small enough that the number of PHEVs accessing or leaving the tagged distribution grid per slot is typically no larger than one. The objective of the problem is to maximize the total number of PHEVs that can be charged under the given system capacity by optimizing the charging scheduling vectors

$$
X(t)=\left[X_{1}(t), X_{2}(t), \ldots, X_{N}(t)\right], \quad \forall t=1,2, \ldots, T,
$$

where $X_{k}(t) \in\{0,1\}, \quad \forall k=1,2, \ldots, N, N$ is the total number of PHEVs and $T$ is the total time slots.

The scheduling needs to consider three constraints. First, each PHEV cannot wait longer than the maximum tolerable delay. Second, the load of each bus cannot exceeds its capacity. Third, the voltage drop of any bus cannot be larger than the maximum allowed voltage drop at any time. This problem can be formulated as follows:

$$
\begin{gathered}
\max \quad N \\
\text { subject to: } \omega(k) \leq \omega_{m}(k), \\
f_{i}\left(X(t), L_{\text {base }}^{i}(t)\right) \leq 1, \quad \forall i=1,2, \ldots, M, \\
f_{v}\left(X(t), L_{\text {base }}^{i}(t)\right) \leq V_{m i}, \quad \forall i=1,2, \ldots, M,
\end{gathered}
$$

where $M$ is the number of buses in the distribution grid; $\omega(k)$ is the total waiting time of PHEV $k ; \omega_{m}(k)$ is the maximum tolerable delay time of PHEV $k ; V_{m i}$ is the maximum allowed voltage drop of bus $i ; L_{\text {base }}^{i}$ is the base load on bus $i ; f_{i}$ is the mapping function that calculates the loading rate from all the load; $f_{v}$ is the mapping function that calculates the voltage drop from all the load.

Since it is an integer optimization problem, which is difficult to obtain the optimal solution in polynomial time, and constraints (4) and (5) are not linear, in this paper, we aim to obtain a sub-optimal solution in a distributed and real-time manner and compare its performance with the best results using exhaustive search in simulation.

\section{Proposed Framework}

The proposed framework includes three entities: a control center covering one or more medium voltage grids, one smart agent per house, and PHEVs. It is important to note that although our proposed framework is used for PHEV charging, other elastic load such as washing machine, water heater, and air conditioner may also be applicable under this framework to provide demand response.

\section{A. Control Center}

The control center in the grid will monitor two kinds of information: load and voltage drop. All the information can be obtained from sensors distributed in the grid. In this paper, we assume that an existing smart grid communication infrastructure is available to connect the control center, sensors and smart agents, and the communication delay and packet losses are negligible [16], [17], [21]. We assume that the data from all the sensors are updated in real-time. The influence of grid information update delay on system performance will be discussed in Section VIII.

From both the historical data and grid topology information, the control center is able to determine which buses are more likely to experience the congestion or voltage drop problems. These are called critical buses, which constitute the congested bus set $S_{c}$ and voltage drop bus set $S_{v}$.

When a PHEV is plugged in, the smart agent which makes scheduling decisions for the PHEV will request a data set from the control center including parameters relevant to the critical buses. The data set contains the loading rate of the most easyto-congest bus affected by that PHEV (denoted as $\gamma_{c}$ ), and a voltage ratio $\gamma_{v}$.

At time slot $t$, the loading rate of bus $i$ is defined as follows (to simplify the notation, we drop $t$ in the following equations):

$$
\gamma_{c}(i)=\frac{\text { current load of bus } \mathrm{i}}{\text { maximum allowed load of bus } \mathrm{i}} .
$$

Then $\gamma_{c}$ is obtained by choosing the maximum rate of the bus from $S_{c}$, which is the most vulnerable to the congestion problem:

$$
\gamma_{c}=\max \gamma_{c}(i), \quad i \in S_{c}
$$

Similarly $\gamma_{v}$ is obtained from all affected buses:

$$
\begin{gathered}
\gamma_{v}(i)=\frac{\text { current voltage drop of bus } \mathrm{i}}{\text { maximum tolerable voltage drop of bus } \mathrm{i}} . \\
\gamma_{v}=\max \gamma_{v}(i), \quad i \in S_{v} .
\end{gathered}
$$

For example, from [5], for charging load under all the MV/LV transformers, bus A in Fig. 1 suffers the severest congestion problem and bus 1 may experience the largest voltage drop. If a PHEV at location $L$ is plugged in, the smart agent at $L$ will request the grid information from the control center which will put $\gamma_{c}(A)$ as $\gamma_{c}$ and $\gamma_{v}(1)$ as $\gamma_{v}$ into a data set and then deliver it to the smart agent.

Meanwhile, the control center will keep monitoring the status of the whole power grid and send instructions to the smart agents to adjust some control parameters which will affect their scheduling decisions if necessary. We will discuss these parameters and actions in Section VI.

\section{B. Smart Agents}

The smart agents (or the smart meters) can schedule PHEVs charging. The scheduling decisions are made based on the data sets received from the control center and the algorithms described in Section VI. 
All the houses adopting our algorithms will receive incentive from the electricity company depending on the contributions they make. (How to determine the contribution and design an incentive mechanism is left for future research.)

It is worth to notice that the user can always let the smart agent charge the PHEV without waiting, in this case the PHEV becomes critical load and the user will not receive compensation from the power company.

\section{PHEV}

In this paper, we assume that the PHEVs are plugged in as soon as they arrive home. The departure time can either be set by the user or by the smart agent according to the historical data. Then the smart agent can calculate the maximum tolerable delay time $\left(\omega_{m}\right)$ for PHEV charging based on the total parking time (the time between the departure and arrival, denoted by $\omega_{t}$ ), state of charge (SOC) and charging power $\left(P_{c}\right)$. Specifically, $\omega_{m}=\omega_{t}-(1-S O C) P_{B} / P_{c}$, where $P_{B}$ is the battery capacity. Here we use a linear battery model which is the same as the model used in [2], [9], [15]. In a real system, the PHEV charging behavior can be more complicated. Our algorithm is still applicable so long as the smart agent knows how much time is needed to charge the PHEV and the total parking time of the PHEV.

To satisfy users' requirements, the smart agent should guarantee that the total delay is always less than the maximum tolerable delay time. In addition, to maintain fairness, some charging PHEVs may terminate charging in the middle to yield the charging opportunity to other waiting ones. The smart agents will also assign a higher priority to those PHEVs which have waited for a longer time.

\section{RAndom Access Algorithm Design}

The design objective of our algorithm can be summarized as follows. First of all, to avoid bus congestion and voltage drop problems in the distribution power grid, (4) and (5) should be satisfied. Second, fairness should be maintained among all the PHEVs. Third, users' preferences should be taken into consideration. Fourth, (3) should be satisfied so that all the PHEVs can be fully charged before their departure. Finally, this algorithm should not rely on future load prediction and is simple enough to be executed online.

The flow chart of our proposed algorithm is presented in Fig. 2. Fig. 2 (a) describes how the smart agent schedules PHEV charging based on the received information, while Fig. 2 (b) shows the process of providing demand response to protect the distribution grid. We will cover the design details in the following subsections. To meet the first design objective, when a PHEV is plugged in, the smart agent will calculate all the access probabilities based on the data set received from the control center and choose the minimum one to decide the charging probability.

Specifically, from $\gamma_{c}$, and $\gamma_{v}$, the access probability $p_{1}\left(\gamma_{c}\right)$ and $p_{1}\left(\gamma_{v}\right)$ can be obtained using the algorithm described below respectively. Then $p_{1}$ is set to be the minimum one to decide the charging probability of the PHEV.

$$
p_{1}=\min \left\{p_{1}\left(\gamma_{c}\right), p_{1}\left(\gamma_{v}\right)\right\} .
$$

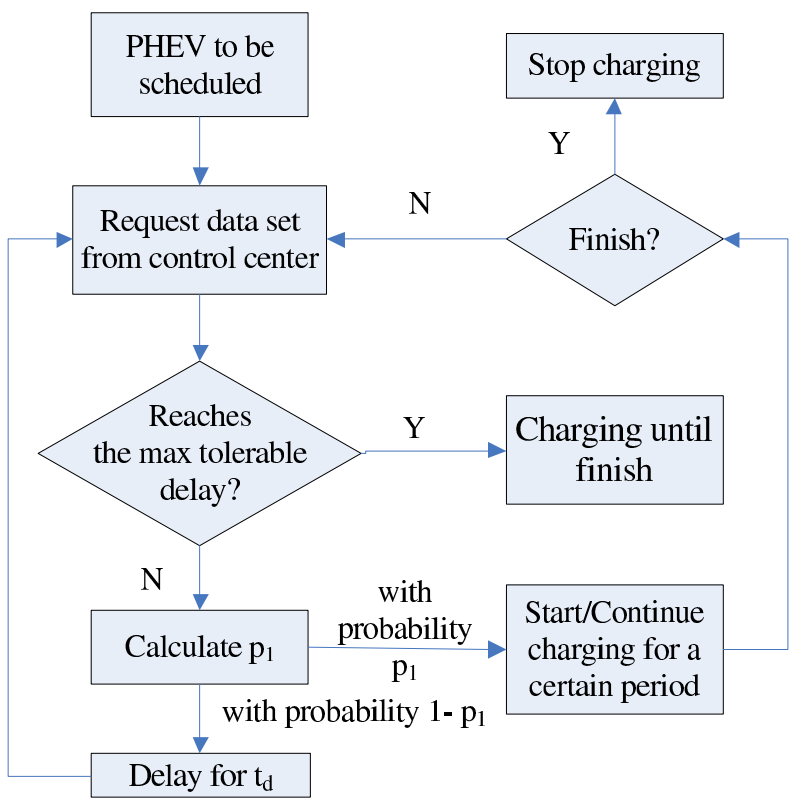

(a) Schedule PHEV charging

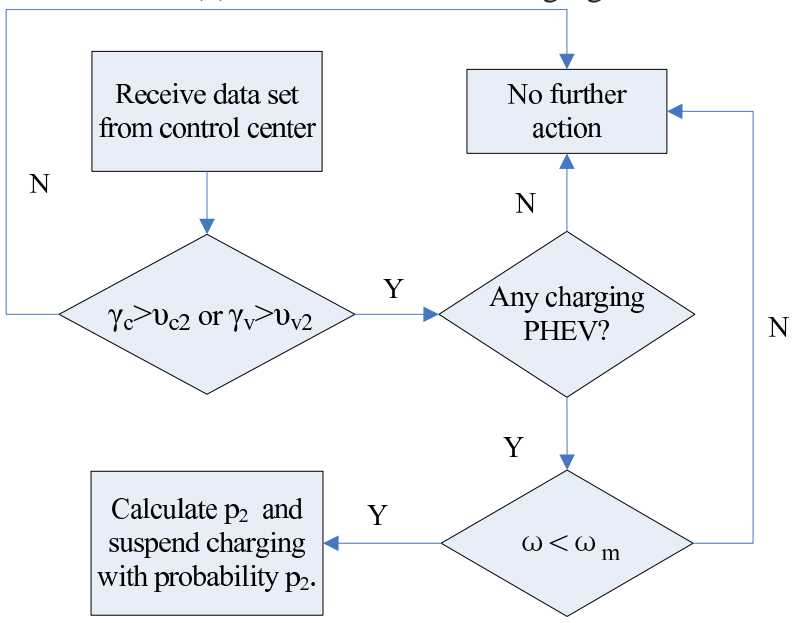

(b) Demand Response

Fig. 2. Flow chart for smart agent

The access probability is calculated as follows. If both of the received ratios $\gamma_{c}$ and $\gamma_{v}$ are below the corresponding threshold one (ts1), the PHEV will start charging with probability one; otherwise, if any of these ratios is higher than its corresponding ts 1 , the charging of the PHEV is restricted based on a back-off algorithm: with probability $p_{1}$, this PHEV will start charging immediately; with probability $1-p_{1}$, it will be delayed by $t_{d}$ and then try again. If any of the ratios is even higher than the corresponding threshold two (ts2), the charging probability $p_{1}$ is set to zero unless the PHEV reaches its maximum tolerable delay time. In addition, in each time slot, the control center will broadcast the ratio when it is larger than the corresponding ts2, to notify all the relevant smart agents until this ratio falls below ts 2 again. These smart agents, upon receiving the notification, will suspend the charging PHEVs with a probability $p_{2}$ to protect the distribution grid. By stopping charging PHEVs every time slot based on a probability, a fast demand response can be achieved. 
To provide an equal chance for each PHEV, every smart agent will acquire the data sets from the control center again after the PHEV has been charged for a period of time and then decide whether to let it continue charging or to suspend it based on the updated probability calculated again from the received ratios. This will provide an opportunity for other waiting PHEVs to start charging. All the waiting PHEVs will obtain a higher priority to charge with a larger waiting time. A suitable charging period is selected to protect the batteries.

However, when a PHEV reaches its maximum tolerable delay time, the smart agent will let it start charging immediately to meet the design objective four.

In the following part, we describe how to design the access probability $p_{1}$ and the suspending probability $p_{2}$ considering bus congestion and voltage drop in the distribution grid.

\section{A. Bus Load Congestion}

Obviously, the access probability $p_{1}\left(\gamma_{c}\right)$ should be close to 1 if the ratio $\gamma_{c}$ only exceeds ts 1 slightly and it must decrease fast when $\gamma_{c}$ approaches ts 2 . Therefore, $p_{1}\left(\gamma_{c}\right)$ is designed as an exponential function:

$$
p_{1}\left(\gamma_{c}\right)= \begin{cases}\kappa_{1} e^{-\alpha_{c}\left(\gamma_{c}-\nu_{c 1}\right)+\beta_{c} \omega / \omega_{m}}+\delta_{1}, & \text { if } \omega<\omega_{m}, \\ 1, & \text { if } \omega=\omega_{m},\end{cases}
$$

where $\omega$ is the current waited time of the tagged PHEV, $\omega_{m}$ represents its maximum tolerable delay, $\nu_{c 1}$ is the value of threshold one for bus congestion, the parameter $\delta_{1}$ is used to reflect user's preference, and the global parameter $\kappa_{1}$ is used by the control center for global adjustment if needed.

In the above design, $p_{1}\left(\gamma_{c}\right)$ decreases exponentially when $\gamma_{c}$ increases, which will restrict the number of PHEVs start charging when the bus congestion level is high. Since the smart agent will use the largest $\gamma_{c}(i)$ to calculate the access probability, all the critical buses can be protected.

The global parameter $\kappa_{1}$ can be set by the control center through notification messages and it is the same for all the smart agents. By decreasing $\kappa_{1}$, the probability to start charging is decreased, so the demand caused by PHEV charging is reduced when $\gamma_{c}$ is between ts 1 and ts2, and vice versa. By default, $\kappa_{1}$ is set to 1 .

The parameter $\alpha_{c}$ determines how fast $p_{1}\left(\gamma_{c}\right)$ will decrease when the current loading rate $\gamma_{c}$ approaches threshold two for bus congestion (with value $\nu_{c 2}$ ). Considering the design objective, we define $p_{1}\left(\nu_{c 1}\right)=1$ and $p_{1}\left(\nu_{c 2}\right)=\varepsilon$ when $\omega<$ $\omega_{m}$ where $\varepsilon$ is a very small positive number. Then, $\alpha_{c}$ can be expressed as follows:

$$
\alpha_{c}=\frac{\ln \varepsilon}{\nu_{\mathrm{c} 1}-\nu_{\mathrm{c} 2}} .
$$

On the other hand, this PHEV charging request may also be delayed with probability $1-p_{1}$. If $\omega_{m}-\omega>t_{m}$, then the delay time $t_{d}$ is randomly selected from $\left[0, t_{m}\right]$, where $t_{m}$ is the upper bound for the delay; otherwise, $t_{d}$ is set as $\omega_{m}-\omega$.

From (11), PHEVs with the waiting time closer to $\omega_{m}$ have a higher probability to access the grid. This will maintain delay and fairness among all the PHEVs. The maximum tolerable waiting time $\omega_{m}$ can be set by the user or by the smart agent based on the historical data. Here we assume that an incentive mechanism is used so all the users set an appropriate $\omega_{m}$ according to their real needs. The parameter $\beta_{c}$ is used to determine the weight of the waiting time on the charging probability. The value for $\beta_{c}$ can be defined as follows: let $p_{1}\left(\nu_{c 2}\right)=\rho, \kappa_{1}=1$ and $\delta_{1}=0$ in (11), and we have: $p_{1}\left(\nu_{c 2}\right)=e^{-\alpha_{c}\left(\nu_{c 2}-\nu_{c 1}\right)+\beta_{c}}=\rho \Rightarrow \beta_{c}=\ln \rho+\alpha_{\mathrm{c}}\left(\nu_{\mathrm{c} 2}-\nu_{\mathrm{c} 1}\right)$.

To fully utilize PHEV's delay time, $\rho$ should be less than 1 ; otherwise, every PHEV will get a high probability to start charging before it can be delayed to its maximum tolerable delay time even when $\gamma_{c}$ is high. According to our simulation, $\rho$ can be set between 0.3 and 0.6 empirically.

When $\gamma_{c}$ is greater than ts2, demand response mechanism will take place. The probability to suspend a charging PHEV is denoted as $p_{2}\left(\gamma_{c}\right)$. Similarly, $p_{2}\left(\gamma_{c}\right)$ is also designed as an exponential function:

$$
p_{2}\left(\gamma_{c}\right)= \begin{cases}\kappa_{2} e^{\lambda_{c}\left(\gamma_{c}-1\right)}+\delta_{2}, & \text { if } \omega<\omega_{m} \\ 0, & \text { if } \omega=\omega_{m}\end{cases}
$$

where $\kappa_{2}$ is another global parameter set by the control center to adjust demand response speed. $\delta_{2}$ is used to represent users' preferences, similar to $\delta_{1}$. Both $\delta_{1}$ and $\delta_{2}$ can be set by the user or learnt by the smart agent. Of course, they will be included into the incentive mechanism aimed to determine users' contributions.

The parameter $\lambda_{c}$ determines the increasing speed of $p_{2}\left(\gamma_{c}\right)$ when $\gamma_{c}$ is above ts2. If we let $p_{2}\left(\nu_{c 2}\right)=\varepsilon$ and $p_{2}(1)=$ 1 , where $\varepsilon$ is a very small positive number, then $\lambda_{c}$ can be expressed as:

$$
\lambda_{c}=\frac{\ln \varepsilon}{\nu_{\mathrm{c} 2}-1} .
$$

If a PHEV is delayed or suspended, it will try to access the grid again after $t_{d}$ slots. Whether its charging request will be approved depends on the ratio at that time.

\section{B. Voltage Drop}

The influence of a charging PHEV on the voltage of a bus is related to the location of that PHEV. For example, in Fig. 1, the voltage drop of bus 1 is related to every charging PHEV connected to the buses from the feeder point to bus 1 . With the same charging power, the closer the PHEV is to bus 1 , the higher the influence it will be. Therefore, the PHEV at location $L$ has a higher effect on the voltage drop of bus 1 than that at location Y. Consequently, we add a weight of location, $\phi$, to design the access probability function:

$$
p_{1}\left(\gamma_{v}\right)= \begin{cases}\frac{\kappa_{3}}{\phi} e^{-\alpha_{v}\left(\gamma_{v}-\nu_{v 1}\right)+\beta_{v} \omega / \omega_{m}}+\delta_{3}, & \text { if } \omega<\omega_{m} \\ 1, & \text { if } \omega=\omega_{m}\end{cases}
$$

where $\kappa_{3}, \alpha_{v}, \beta_{v}$ and $\delta_{3}$ are set similar to $\kappa_{1}, \alpha_{c}, \beta_{c}$ and $\delta_{1}$ respectively. In practice, it is difficult to define an optimal $\phi$ as it needs perfect grid information. It can be approximated as the ratio of the distance between the PHEV and the feeding point over the distance between the considered critical bus and the feeding point. We will further discuss it in Section VIII. 
Similarly, the suspending probability $p_{2}\left(\gamma_{v}\right)$ can be designed as follows:

$$
p_{2}\left(\gamma_{v}\right)= \begin{cases}\kappa_{4} \phi e^{\lambda_{v}\left(\gamma_{v}-1\right)}+\delta_{4}, & \text { if } \omega<\omega_{m} \\ 0, & \text { if } \omega=\omega_{m}\end{cases}
$$

where $\kappa_{4}, \lambda_{v}$ and $\delta_{4}$ are set similar to $\kappa_{2}, \lambda_{c}$ and $\delta_{2}$ respectively. Therefore, PHEVs closer to the feeding point have a smaller suspending probability for providing demand response.

From the descriptions above, PHEVs are scheduled and charged in a distributed manner, and no prediction is needed. Therefore, the last design objective is met.

Finally, since the smart agent will choose the charging probability calculated from the received data set based on (10) to charge the connected PHEV, all the distribution grid components affected by that PHEV are protected.

\section{PERFormance ANALYSis}

In this section, the capacity of the proposed algorithm on bus congestion is analyzed using a probabilistic method. The performance analysis on voltage drop is similar and omitted due to the space limitation. To simplify the analysis, we assume the maximum tolerable delay of any PHEV is infinity, and the global control parameters $\kappa_{1}, \kappa_{2}$ are set to one. Since Poisson process is an acceptable model if the occurrences are uniformly and independently distributed on an interval of time, the PHEV arriving process is assumed as a Poisson process with an average arrival rate of $\lambda$.

In the first part, we will analyze the performance of the proposed algorithm with real-time grid information, and then we will consider the situation when real-time grid information is not available.

\section{A. Control Center with Real-time Grid Information}

According to the assumption that real-time grid information is available and the slot duration is sufficiently small in Section $\mathrm{IV}$, the number of charging PHEVs will increase or decrease at most by one in each slot. Let us consider a certain critical bus $\mathrm{A}$, assume that the maximum loading rate increase caused by one PHEV on bus A is $\Delta_{m}$. From (11), the maximum probability that the loading rate will exceed ts 2 caused by one arrival PHEV is

$$
p_{1}\left(\nu_{c 2}-\Delta_{m}\right)=e^{-\alpha_{c}\left(\nu_{c 2}-\Delta_{m}-\nu_{c 1}\right)} .
$$

Since demand response will start when $\gamma_{c}>\nu_{c 2}$, this probability should be smaller than a threshold $p_{e}$, where $p_{e}$ is defined by the control center. Let $p_{1}\left(\nu_{c 2}-\Delta_{m}\right) \leq p_{e}$, we can obtain the minimum value of the gap between $\nu_{c 1}$ and $\nu_{c 2}$ :

$$
\nu_{c 2}-\nu_{c 1} \geq-\frac{1}{\alpha_{c}} \ln p_{e}+\Delta_{m}
$$

Define $D_{m}$ the maximum loading rate increase caused by the variation of the base load in one slot. Since the charging probability equals 0 when $\gamma_{c}>\nu_{c 2}$, to prevent the loading rate from exceeding one, the gap between $\nu_{c 2}$ and one should be greater than $D_{m}$ plus $\Delta_{m}$. Then we have

$$
\nu_{c 2} \leq 1-D_{m}-\Delta_{m} .
$$

Besides, once the loading rate exceeds ts2, the probability to suspend charging PHEVs should be less than a pre-defined threshold $p_{t}$ so that only a small number of PHEVs are suspended. $p_{t}$ is set by the control center based on the estimated number of charging PHEVs in the system.

$$
p_{t} \geq p_{2}\left(\nu_{c 2}+\Delta_{m}+D_{m}\right)=e^{\lambda_{c}\left(\nu_{c 2}+\Delta_{m}+D_{m}-1\right)} .
$$

After manipulation, we can obtain another bound for $\nu_{c 2}$.

$$
\nu_{c 2} \leq \frac{1}{\lambda_{c}} \ln p_{t}+1-\Delta_{m}-D_{m}
$$

From (18), (19) and (21), we can obtain the upper bounds of $\nu_{c 1}$ and $\nu_{c 2}$.

\section{B. Control Center Without Real-time Grid Information}

However, there is always a time interval between the information update from sensors to the control center in practice. To analyze the performance of the proposed algorithm in a more practical situation, we assume the grid information is updated every $t$ seconds, and the maximum PHEV arrival rate does not exceed $\lambda$ during the following $t$ seconds.

The expected number of new PHEVs bus A can support without exceeding ts 2 is:

$$
m_{1}=\left\lfloor\frac{\nu_{c 2}-\gamma_{c}}{I_{c}}\right\rfloor,
$$

where $I_{c}$ is the average loading rate increase caused by one PHEV. We further consider the following three situations.

(1) $\gamma_{c}$ is below ts1. All the arrival PHEVs will start charging with probability 1 . The probability that the number of arriving PHEVs does not exceed $m_{1}$ during $t$ is:

$$
P\left(n \leq m_{1}\right)=\sum_{k=0}^{m_{1}} \frac{e^{-\lambda t}(\lambda t)^{k}}{k !} .
$$

(2) $\gamma_{c}$ is between $\nu_{c 1}$ and $\nu_{c 2}$. The probability for each arriving PHEV to start charging is

$$
p_{1}(i)=e^{-\alpha_{c}\left(\gamma_{c}-\nu_{c 1}\right)} .
$$

The probability that the number of arriving PHEVs is less than or equal to $m_{1}$ is

$$
P_{a}=\sum_{k=0}^{m_{1}} \frac{e^{-\lambda t}(\lambda t)^{k}}{k !} .
$$

The probability that the number of PHEVs starting charging is less than $m_{1}$, given the number of arriving PHEV is larger than $m_{1}$, is

$$
P_{b}=\sum_{k=m_{1}+1}^{+\infty} \frac{e^{-\lambda t}(\lambda t)^{k}}{k !} \cdot\left(\sum_{i=0}^{m_{1}}\left(\begin{array}{l}
k \\
i
\end{array}\right) p_{1}{ }^{i}\left(1-p_{1}\right)^{k-i}\right) .
$$

The probability that the total new charging PHEVs' number does not exceed $m_{1}$ is

$$
P\left(n \leq m_{1}\right)=P_{a}+P_{b} .
$$

(3) $\gamma_{c}$ is larger than or equal to $\nu_{c 2}$. In this case, all the arriving PHEVs will not start charging, so their arrivals will not affect the bus load. 
From (23) and (27) we can obtain the probability to exceed $\nu_{c 2}$ for any $\gamma_{c}<\nu_{c 2}$. Similarly, the number of new PHEVs this bus can support without exceeding the bus capacity is

$$
m_{2}=\left\lfloor\frac{1-\gamma_{c}}{I_{c}}\right\rfloor,
$$

Substituting $m_{1}$ by $m_{2}$ in (23), (25), (26), (27), we obtain the probability that the loading rate does not exceed one.

Given $\lambda$ and that the probability to exceed ts 2 and one should below $p_{e}$ and $p_{s}$ respectively, we can obtain the upper bound for ts 1 and ts 2 by using a reversed process. We omit the details due to the space limitation.

It is worth to mention that $\nu_{c 1}$ and $\nu_{c 2}$ can be set dynamically through broadcasting instructions from the control center to all the users according to the changing PHEV arriving rate in different time periods.

On the other hand, given $\nu_{c 1}, \nu_{c 2}, p_{e}, p_{s}$, we can obtain the maximum arrival rate $\lambda$ that the distribution grid can support using the proposed algorithm, which is the capacity of the system.

\section{ViII. Performance Evaluation}

The objective of this simulation is to verify the impact of the proposed control algorithm on bus congestion, and bus voltage drop. The simulation is mainly based on real data with an approximated maximum average arriving rate of 30 PHEVs per minute. The maximum tolerable probabilities to exceed threshold two and bus loading rate one/maximum allowed voltage drop are set to $10^{-2}$ and $10^{-6}$, respectively. $p_{t}$ is set to 0.05 . Whenever the ratio of a critical bus exceeds ts2, the specific ratio of the bus is multicasted to all relevant smart agents every time slot. The situation when the grid information is updated every 60 seconds is also considered in Section VIII-C.

In the following subsections, we only consider one problem at one time under the proposed framework, and assume $p_{1}$ equals $p_{1}\left(\gamma_{c}\right)$ and $p_{1}\left(\gamma_{v}\right)$ in Section VIII-A and Section VIII-B respectively. We compare the performance of the proposed random access (RA) algorithm with that obtained from exhaustive search (ES). For exhaustive search, we choose the one with the minimum average PHEV waiting time so it can result in the maximum number of PHEVs being supported without violating the constraints. In the exhaustive search, we assume that PHEVs' charging can be interrupted at any time and any frequency; therefore, ES result can also be considered as the performance upper bound. Similar to [27], we do not consider reactive power or grid losses as they will make the simulation time much longer. On the other hand, in a real system, as the smart agents use the measured data, which reflects the reactive power and grid losses, etc., to calculate the access probabilities, our algorithm can still be applicable.

\section{A. Bus Load Congestion}

According to the simulation results in [5], bus A suffers the severest congestion problem so its loading rate is delivered to the downstream smart agents when required. In this

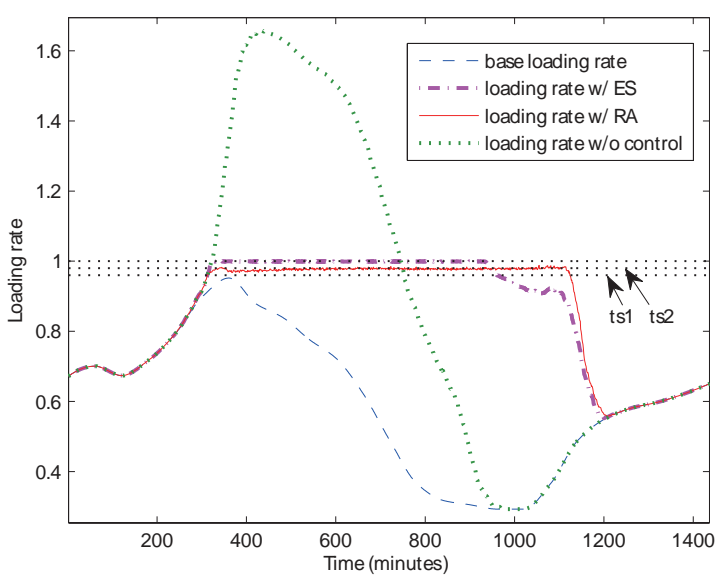

(a) Loading rate variation

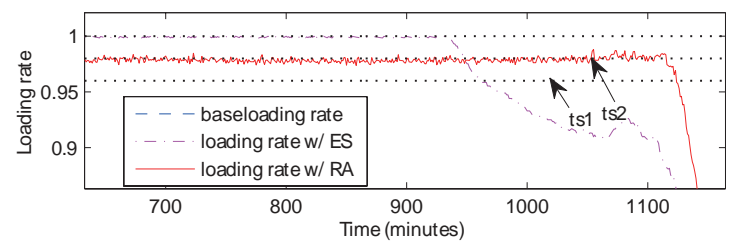

(b) Zoom-in of (a)

Fig. 3. Loading rate of bus A with 742 PHEVs

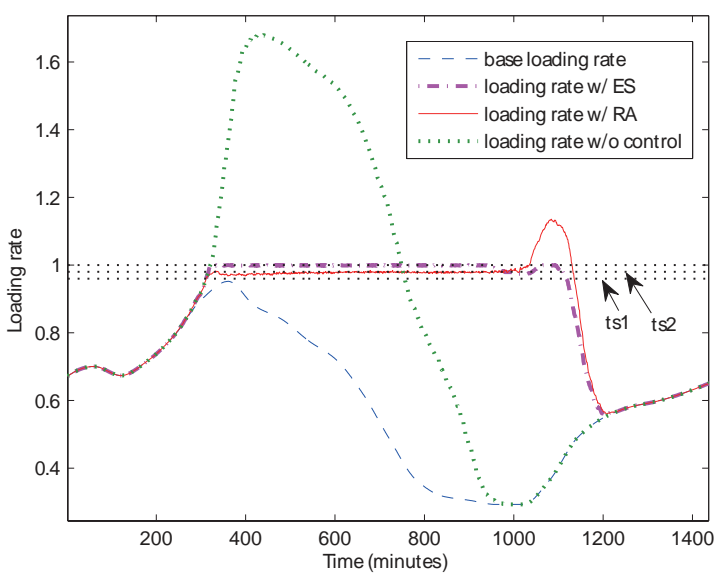

Fig. 4. Loading rate of bus A with 765 PHEVs

simulation, $\nu_{c 1}$ and $\nu_{c 2}$ are set to 0.96 and 0.98 respectively according to the analysis in Section VII.

Fig. 3 (a) illustrates the loading rate of bus A with 742 PHEVs. To view the curves more clearly, the most critical time period is zoomed in and shown in Fig. 3 (b). This can be considered as the capacity of the proposed algorithm because the loading rate reaches ts 2 at the end of the charging period. As shown in the figure, our proposed algorithm can flatten the bus loading rate quite well while the uncontrolled loading rate exceeds one by about $70 \%$.

Fig. 4 shows the situation when there are 765 PHEVs which is also the maximum number of PHEVs this distribution grid can support using exhaustive search. Since PHEVs begin to charge immediately whenever they reach their maximum 


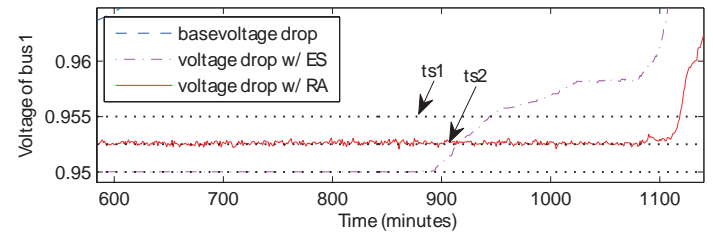

Fig. 5. Voltage drop of bus 1 with grid topology information

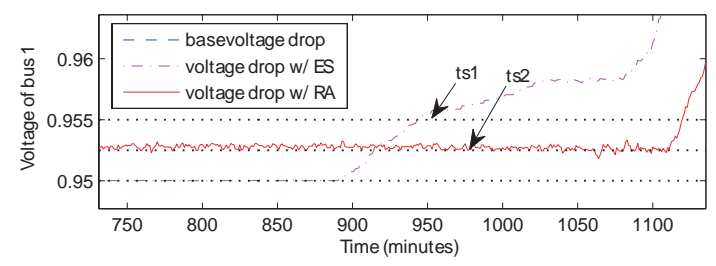

Fig. 6. Voltage drop of bus 1 without grid topology information

tolerable delay time, the loading rate of RA algorithm exceeds one at the end. Through multiple simulations with different PHEV arriving/departure time, exhaustive search with perfect future information can support about 3\% more PHEVs than the proposed algorithm on average in the scenario described in Section III. In other words, the performance of the RA algorithm is very close to the performance upper bound, meanwhile satisfying all the design objectives.

\section{B. Voltage Drop}

In [5], bus 1 has the largest voltage drop, so its voltage ratio is passed down to all the relevant users whenever required. In this simulation, the maximum tolerable voltage drop is $5 \%$, $\nu_{v 1}$ and $\nu_{v 2}$ are set to 0.90 and 0.95 , respectively.

Figs. 5 and 6 show the zoomed in simulation results with and without the grid topology information, respectively. The maximum number of PHEVs the distribution system can support are 730 and 727 on average, respectively. Both of the two cases can restrict the voltage drop near ts 2 . Therefore, we may find that the performance of the random access algorithm is not sensitive to the weight $\phi$ in (15).

\section{Non-real-time Data}

In this part, we explore the performance of our algorithm considering that all the data received at the control center is updated every minute. The value for ts 1 is decreased to 0.95 based on the algorithm in Section VII.

Fig. 7 shows the main part of the loading rate of bus A with 717 PHEVs when non-real-time bus loading rate is delivered to smart agents. This can be considered as the capacity of the

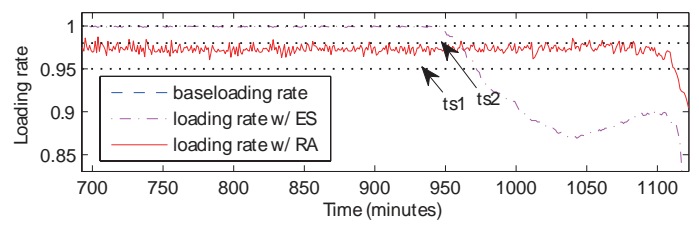

Fig. 7. Loading rate of bus A with 717 PHEVs: non-real time distribution grid using the RA algorithm with non-real time information. Obviously, the controlled loading rate using the RA algorithm fluctuates more severely. The loading rate even exceeds ts 2 several times after minute 700 . The reason is that the number of arrival PHEVs varies greatly during the oneminute time interval, and many PHEVs become critical load when reach their maximum tolerable delay time. Nevertheless, the demand response mechanism suspends some charging PHEVs when the loading rate is over $\nu_{c 2}$ and keeps the loading rate under one all the time. The situation for voltage drop is similar and is omitted due to the space limitation.

\section{CONCLUSION}

In this paper, a random access framework has been proposed to coordinate PHEV charging to maximize the number of PHEVs that can be supported considering bus load congestion and voltage drop constraints. Through the simulation on a residential area distribution grid, it has been demonstrated that our algorithm can achieve the performance with a small gap to the best solution. Besides, it can provide demand response efficiently. We also tested our algorithm when real-time grid information is not available, and the result is only about $6 \%$ worse than the best one. In addition to regulating PHEV charging, the proposed solution can also be applied to other elastic devices.

In this work, the locations of customers will lead to different levels of congestions to the network and thus affect the access probabilities of their PHEVs. This may lead to a fairness problem and requires further study together with other pricing and economic policies. In addition, the distribution grid in this paper has a radial topology, for a meshed distribution grid, things are much more complicated. However, the main idea of the proposed algorithm is still useful to control the PHEV access if the critical bus is congested. These are important further research issues. In practice, several other problems in the distribution grid should be considered, such as medium voltage to low voltage (MV/LV) transformer overload. How to make a tradeoff between the complexity of the control algorithm and the distribution grid efficiency is left for future research.

\section{REFERENCES}

[1] S. Letendre and R. A.Watts, "Effects of plug-in hybrid electric vehicles on the vermont electric transmission system," in Proc. Transp. Res. Board Annu. Meet., Washington, D.C, Jan, 2009.

[2] D. Wu, D. Aliprantis, and L. Ying, "Load scheduling and dispatch for aggregators of plug-in electric vehicles," IEEE Transactions on Smart Grid, vol. 3, no. 1, pp. 368-376, March 2012.

[3] J. Taylor, A. Maitra, M. Alexander, D. Brooks and M. Duvall. "Evaluations of the impact of plug-in electric vehicle on distribution system operations," PES 09, July 2009.

[4] Ahmad Karnama, "Analysis of integration of plug-in hybrid electric vehicles in the distribution grid," M.S.thesis, Stockholm, Sweden 2009.

[5] J.A.P. Lopes, F.J. Soares, P.M.R. Almeida, "Identifying management procedures to deal with connection of electric vehicles in the grid," IEEE PowerTech, June 2009.

[6] O. Sundstrom, and C. Binding, "Flexible charging optimization for electric vehicles considering distribution grid constraints," IEEE Transactions on Smart Grid, vol.3, no.1, pp. 26-37, March 2012.

[7] O. Sundstrom, and C. Binding, "Planning electric-drive vehicle charging under constrained grid conditions," POWERCON 2010, 24-28 Oct. 2010. 
[8] S. Shao, M. Pipattanasomporn and S. Rahman, "Challenges of PHEV penetration to the residential distribution network," IEEE Power and Energy Society General Meeting, 26-30 Jul. 2009.

[9] S. Shao, M. Pipattanasomporn and S. Rahman, "Demand response as a load shaping tool in an intelligent grid with electric vehicles," IEEE Transactions on Smart Grid, Dec 2011.

[10] S. Shao, M. Pipattanasomporn and S. Rahman, "Grid integration of electric vehicles and demand response with customer choice," IEEE Transactions on Smart Grid, March 2012.

[11] USA, "Summary of travel trends: 2009 national household travel survey," Federal Highway Administration, 2012, [Online]. Available: http://nhts.ornl.gov/2009/pub/stt.pdf

[12] RELOAD Database Documentation and Evaluation and Use in NEMS, [Online]. Available: http://www.onlocationinc.com/ LoadShapesReload2001.pdf.

[13] National Energy Modeling System (NEMS), [Online]. Available: http: //www.eia.gov/oiaf/aeo/overview/.

[14] S. Shao, T. Zhang, M. Pipattanasomporn and S. Rahman, "Impact of TOU rates on distribution load shapes in a smart grid with PHEV penetration," IEEE PES, Apr. 2010.

[15] H. Liang, B. Choi, W. Zhuang, and X. Shen, "Optimizing the energy delivery via V2G systems based on stochastic inventory theory," IEEE Transactions on Smart Grid, vol. 4, no. 4, pp. 2230-2243, Dec. 2013.

[16] L. Zheng, N. Lu, L. Cai, "Reliable Wireless Communication Networks for Demand Response Control," IEEE Transactions on Smart Grid, vol.4, no.1, pp.133-140, March 2013

[17] R. Deng, J. Chen, X. Cao, Y. Zhang, S. Maharjan, S. Gjessing, "Sensingperformance tradeoff in cognitive radio enabled Smart Grid," IEEE Transactions on Smart Grid, vol. 4, no. 1, pp.302-310, March. 2013.

[18] K. Zhou and L. Cai, "A decentralized access control algorithm for PHEV charging in smart grid," Springer Energy Systems, 2013.

[19] U.S. Department of Transportation, Federal Highway Administration, "National household travel database," [Online]. Available: http://nhts. ornl.gov/download.shtml.

[20] Electric Power Research Institute, "Comparing the benefits and impacts of hybrid electric vehicle options," Palo Alto, CA, Tech. Rep. 1000349, Jul. 2001.

[21] Z. Fadlullah, M. Fouda, N. Kato, A. Takeuchi, N. Iwasaki, and Y. Nozaki, "Towards intelligent machine-to-machine communications in Smart Grid," IEEE Communications Magazine, vol. 49, no. 4, pp. 60-65, Apr. 2011.

[22] M. D. Galus, R. A. Waraich, and G. Andersson, "Predictive, distributed, hierarchical charging control of PHEVs in the distribution system of a large urban area incorporating a multi agent transportation simulation," 17th Power Systems Computation Conference, Stockholm Sweden, 2011.

[23] D. Wu, D. C. Aliprantis and K. Gkritza, "Electric energy and power consumption by light-duty plug-in electric vehicles, "IEEE Transactions on Power Systems, vol. 26, NO. 2, MAY 2011.

[24] K. Clement-Nyns, E. Haesen, J. Driesen, "The impact of charging plugin hybrid electric vehicles on a residential distribution grid, " IEEE Transactions on Power Systems, vol. 25, NO. 1, february 2010.

[25] E. Sortomme et. al, "Coordinated charging of plug-in hybrid electric vehicles to minimize distribution system losses," IEEE Transactions on Smart Grid, vol 2, issue 1, March 2011.

[26] R. J. Rei, et. al,"Grid interactive charging control for plug-in electric vehicles," 2010 13th International IEEE Annual Conference on Intelligent Transportation Systems, Madeira Island, Portugal, September 1922, 2010.

[27] P. Richardson, D. Flynn, and A. Keane, "Optimal charging of electric vehicles in low-voltage distribution systems," IEEE Transactions on Power Systems, vol. 27, NO. 1, Feb 2012.

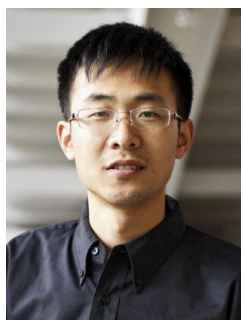

Kan Zhou received the B.S. and M.A.Sc. degree in the Department of Electrical and Computer Engineering from Southeast University, Nanjing, China, in 2008 and 2011 respectively. He is currently working toward the Ph.D. degree in electrical and computer engineering in the University of Victoria, British Columbia, Canada. His fields of interest include PHEV scheduling, smart grid and artificial intelligence.

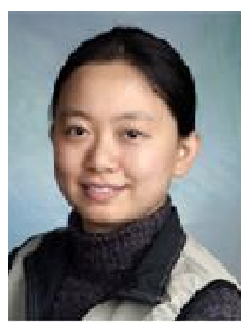

Lin Cai (S'00-M'06-SM'10) received her M.A.Sc. and $\mathrm{PhD}$ degrees (awarded Outstanding Achievement in Graduate Studies) in electrical and computer engineering from the University of Waterloo, Waterloo, Canada, in 2002 and 2005, respectively.

Since 2005, she has been an Assistant Professor and then an Associate Professor with the Department of Electrical \& Computer Engineering at the University of Victoria. Her research interests span several areas in wireless communications and networking, with a focus on network protocol and architecture design supporting emerging multimedia traffic over wireless, mobile, ad hoc, and sensor networks. She has been a recipient of the NSERC Discovery Accelerator Supplement Grant in 2010, the best paper award of IEEE ICC 2008, and the best academic paper award of IEEE WCNC 2011. She has served as a TPC symposium co-chair for IEEE Globecom'10 and IEEE Globecom'13, and the Associate Editor for IEEE Transactions on Wireless Communications, IEEE Transactions on Vehicular Technology, EURASIP Journal on Wireless Communications and Networking, International Journal of Sensor Networks, and Journal of Communications and Networks (JCN). 